Revue internationale P.M.E.

Économie et gestion de la petite et moyenne entreprise

\title{
Innovation et exportation : quelle compatibilité pour les PME ?
}

\section{Alice Guilhon, Bernard Guilhon et Denis Peguin}

Volume 7, numéro 2, 1994

URI : https://id.erudit.org/iderudit/1008390ar

DOI : https://doi.org/10.7202/1008390ar

Aller au sommaire du numéro

Éditeur(s)

Presses de l’Université du Québec

ISSN

0776-5436 (imprimé)

1918-9699 (numérique)

Découvrir la revue

Citer cette note

Guilhon, A., Guilhon, B. \& Peguin, D. (1994). Innovation et exportation : quelle compatibilité pour les PME ? Revue internationale P.M.E., 7(2), 85-103.

https://doi.org/10.7202/1008390ar
Résumé de l'article

L'objectif de cette note de recherche est de s'interroger sur les facteurs qui permettent d'articuler les comportements d'innovation et l'exportation des PME. L'analyse met en évidence le rôle que jouent les investissements intellectuels dans le processus de maturation des entreprises dans leur environnement. De ce point de vue, deux groupes d'entreprises peuvent être distingués : celles qui bouleversent leur organisation globale (lien entre implantation technique, investissement intellectuel et exportation) et celles qui adaptent leur organisation aux nouvelles contraintes de l'environnement (faible intégration technique, investissement intellectuel ponctuel et faible engagement à l'exportation). Ces comportements sont vérifiés par des analyses statistiques effectuées sur un échantillon de PME de la région PACA. 


\title{
Notes de recherche
}

\section{Innovation et exportation : quelle compatibilité pour les PME?}

\author{
Alice GUILHON * \\ Bernard GUILHON** \\ Université d'Aix-Marseille ॥ \\ Denis PEGUIN*** \\ Université de Provence
}

\begin{abstract}
RÉSUMÉ
L'objectif de cette note de recherche est de s'interroger sur les facteurs qui permettent d'articuler les comportements d'innovation et l'exportation des PME. L'analyse met en évidence le rôle que jouent les investissements intellectuels dans le processus de maturation des entreprises dans leur environnement. De ce point de vue, deux groupes d'entreprises peuvent être distingués: celles qui bouleversent leur organisation globale (lien entre implantation technique, investissement intellectuel et exportation) et celles qui adaptent leur organisation aux nouvelles contraintes de l'environnement (faible intégration technique, investissement intellectuel ponctuel et faible engagement à l'exportation). Ces comportements sont vérifiés par des analyses statistiques effectuées sur un échantillon de PME de la région PACA.
\end{abstract}

* Alice Guilhon est docteur en sciences de gestion (octobre 1993), maître de conférences à l'Université d'Aix-Marseille II et membre de l'ERFI à l'Université de Montpellier I.

** Bernard Guilhon est professeur de la Faculté de sciences économiques d'AixMarseille II et directeur du Département d'économie industrielle du CEFI (URA CNRS n 951).

*** Denis Peguin est docteur habilité et maître de conférences à l'Université de Provence où il dirige le DESS «Affaires Internationales». Il est membre du GREQAM-LEQAM. (UMA CNRS n 9990)

Adresse : CEFI, Département d'économie industrielle, Université d'Aix-Marseille II, Château La Farge, Route des Milles, 13290 Les Milles, France. 


\begin{abstract}
The aim of this paper is to identify factors that lead to the compatibility between innovation and export behaviors in a small business firm. Among them intellectual investments play a leading role in the evolution of the firm's environment. From this point of view, two types of firm do exist. The first type is characterized by a disruption in the general organization of the firm with the introduction of new technology, personal improvement programs and export. The second type of firm adopts a progressive strategy with low technological integration, localized intellectual investment and small engagement to export business. Using a sample of small business firms in the French's Provence area, we verify the existence of such strategies.
\end{abstract}

\title{
RESUMEN
}

El objectivo de ese articulo es determinar los elementos que permiten articular las strategias de inovacion y exportacion de las pequenas empresas. Esta analisis pone en relieve la importancias de las inviertos intelectuales en el procesus de maduracion de los empresas con su entoma. Des de este punto de vista se peuden distinguir dos clases de empresas: las que modificon la organizacion global de la impresa (relacion entre introduccion technologica, inviertos intelectuales y exportacion) y las que siguer una strategia que adapta la organizacion con poca integracion technica, inviertos intelectuales punctuales y debil implicacion. Estos comportamientos han sido overiguados por analisis estadicas sobre pequenas empresas de una region francesa (PACA).

\section{Introduction}

Pour s'interroger sur le rôle que peuvent jouer les petites et moyennes entreprises (PME) dans le développement des exportations d'une région, on doit identifier les forces à l'œuvre dans ce type de firmes pour améliorer leur position sur des marchés de plus en plus concurrentiels. Ces forces, pour reprendre les analyses traditionnelles en ce domaine, peuvent provenir soit du développement technologique, soit du développement du marché, à moins que les deux jouent conjointement pour déterminer la position concurrentielle d'une firme sur un marché ${ }^{1}$. En effet, comme le montre une étude récente du SESSI, près de $60 \%$ des entreprises innovantes déclarent être motivées dans leur démarche d'innovation par le marché contre $26 \%$ par la technologie. On peut cependant penser que ces pourcentages varient selon la taille des entreprises. La grande entreprise, ayant une capacité autonome d'innovation (R-D interne) plus grande, sera plus incitée à innover indépendamment des forces du marché que la petite entreprise faisant davantage appel à des sources externes pour ses innovations.

1. C'est la position défendue par M. Porter (1990) lorsqu'il présente son «diamant». 
Il faut cependant se garder de conclure trop vite à une totale soumission de la PME à l'égard du marché pour expliquer sa motivation en matière d'innovation. Il semble, comme nous allons essayer de le montrer dans cette note de recherche, que la logique de fonctionnement de la PME soit incompatible avec cette vision dichotomique des déterminants de l'évolution technologique des firmes. D'une part, comme le soulignent Julien et Jacob (1993), l'innovation technologique dans la PME est considérée comme le moyen de suivre les évolutions du marché. Les entreprises entrent alors dans un processus de «maturation». D'autre part, la $\mathrm{PME}$ se distingue de la grande firme par sa manière de constituer son marché «stratégique». Pour la grande firme, les conditions de production, c'est-à-dire la présence d'économies d'échelle ou d'économies de gamme, est déterminante. La PME, quant à elle, constituera son marché «stratégique» de manière plus pragmatique.

La grande entreprise essaie de segmenter son marché sous la contrainte des conditions de production et, à la limite, de développer des stratégies différenciées selon les segments. Elle peut en particulier substituer un marché extérieur à un marché domestique perdu ou en récession. La PME adopte en général une approche globale à l'égard de son marché stratégique. L'exportation est alors perçue comme le simple prolongement du marché intérieur. La PME cherche à exploiter sur les marchés étrangers l'avantage concurrentiel qu'elle possède déjà sur son marché national. L'extension de son marché vers des contrées plus lointaines, présentant en général des risques plus élevés, relève alors de la même logique que celle qui préside à la constitution du marché de base, c'est-à-dire la logique personnelle du dirigeant (Roux, 1991). Cette observation implique que l'extension du marché pour la PME correspond essentiellement à un acte volontariste de la part du dirigeant-entrepreneur.

Dans notre tentative d'expliquer les déterminants de l'extension du champ d'activités de la PME par l'engagement dans l'exportation, deux arguments viennent en concurrence:

1. L'argument de la taille. De ce point de vue, on ne peut nier le fait - les statistiques sont irréfutables (SESSI, 1990) - que le taux des entreprises exportatrices est croissant avec la taille et que cela est un caractère de différenciation entre la grande et la petite entreprise (Walters et Samlee, 1990).

2. Sans réfuter l'argument de la taille critique (Bricout, 1991), nous proposons un autre point de vue sur les déterminants de l'effort à l'exportation: l'argument de l'approche globale qui caractérise la PME.

Par approche globale, nous entendons le fait que tout changement intervenant dans les procédés de production, le produit ou le marché est appréhendé par la PME de manière globale de telle sorte que les mesures prises impliquent 
une réorganisation d'ensemble de l'entreprise. En conséquence, on cherche à expliciter la logique qui sous-tend cette réorganisation visant principalement les procédés et les modes d'organisation. Pour cela, nous adopterons une définition large de l'innovation qui concerne les techniques, l'organisation et les ressources humaines.

Nous présenterons donc, dans une première section, la problématique et la méthodologie développée pour cette étude. La deuxième section sera consacrée aux résultats d'une enquête effectuée auprès d'un échantillon d'entreprises de la région PACA et portant sur les procédures de mise en place de nouvelles technologies.

\section{Problématique et méthodologie}

La problématique sur laquelle repose ce travail considère que les PME peuvent avoir deux types de comportement en fonction des caractéristiques des marchés et des ressources qu'elles mettent en œuvre. Ces comportements peuvent être repérés par une analyse quantitative dont le contenu est précisé dans le second point.

\subsection{La mise en évidence des stratégies}

Le niveau actuel de turbulence de l'environnement et les transformations de l'acte de produire nécessitent la mobilisation de ressources à la fois matérielles et immatérielles (formation, R-D, communication) (Bouchet, 1989). Cette mobilisation de ressources conduit à définir des stratégies de deux types : l'adaptative et l'anticipative.

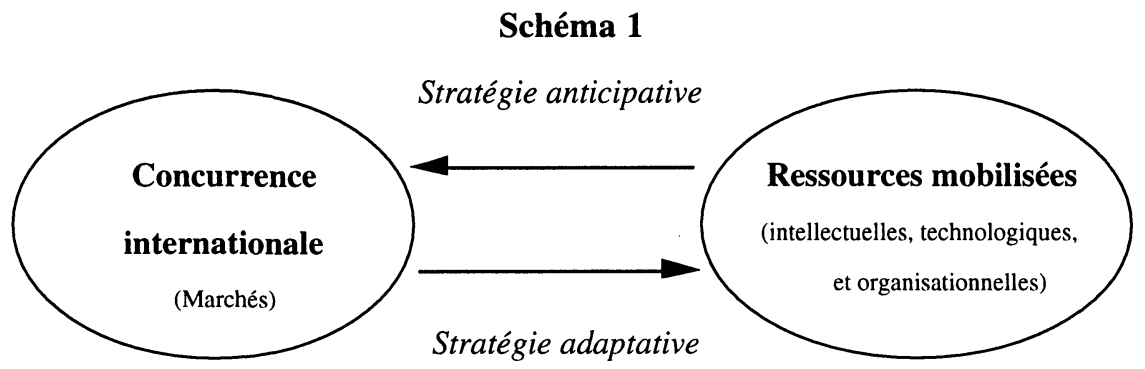

Le domaine d'activité est construit par la firme et accompagne la transformation des structures et des comportements (stratégie anticipative). Dans ce contexte, le dirigeant a un a priori sur le domaine d'activités futur de l'entreprise, il spécifie alors les ressources internes pour atteindre cet objectif. Que l'en- 
semble de l'entreprise soit concerné par le nouveau marché suppose une restructuration ou un bouleversement de l'organisation, c'est-à-dire une redéfinition des produits et un changement dans les méthodes de production et de commercialisation qui ne concerne pas seulement les nouvelles activités. Cet ensemble de transformations introduites par le dirigeant va permettre à l'entreprise de se doter d'un nouveau marché stratégique.

La stratégie anticipative peut être résumée par la séquence suivante:

\section{Schéma 2}

\section{Stratégie anticipative}

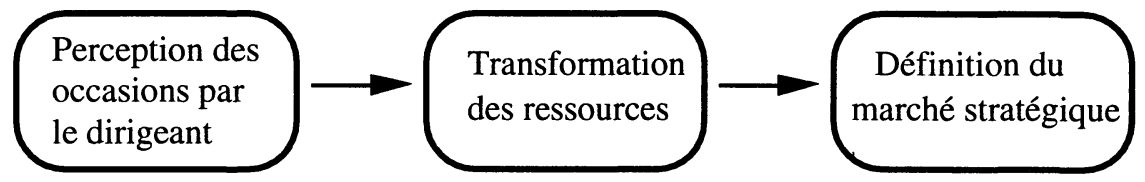

Le domaine d'activités s'impose et véhicule des contraintes d'adaptation progressive des structures et des comportements (stratégie adaptative). C'est selon certaines études, le comportement habituel des PME (Marchesnay, 1990). Elles s'adaptent, pas à pas, aux changements intervenant dans leur environnement. Aucun bouleversement n'est introduit, les transformations se font progressivement. La conséquence en est que des mutations importantes sur le plan technologique ou sur le plan commercial ne peuvent pas être mises en place par la PME. On peut représenter ainsi cette stratégie:

\section{Schéma 3}

\section{Stratégie adaptative}

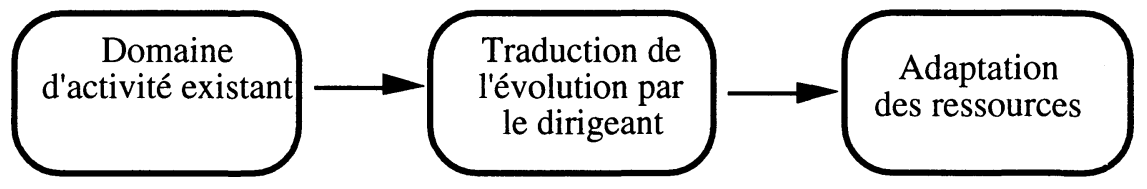

L'énoncé de ces deux stratégies permet de formuler quatre hypothèses de travail.

Hypothèse 1: L'engagement à l'exportation provoque un changement technologique qui porte sur l'ensemble des fonctions de l'entreprise et non pas uniquement sur certains postes de travail. 
Généralement, la PME se distingue de la grande entreprise par le fait que son développement est graduel, c'est-à-dire par étapes et sans grand bouleversement. Par contraste, des ruptures et des seuils caractérisent l'évolution de la grande entreprise, comme par exemple, la réduction de son activité (vente de divisions ou de filiales) ou dans son développement (fusion, délocalisation...). Notre première hypothèse se justifie par le fait que, dans certains cas, un bouleversement de la structure organisationnelle de la PME s'impose. Ainsi, l'engagement d'une PME dans l'exportation nécessitera la mise en place de nouveaux moyens susceptibles de remettre en cause les normes, les politiques et les objectifs de l'entreprise.

Hypothèse 2: Plus l'entreprise développe des activités intégrées (conception, fabrication, commercialisation), plus elle va réaliser des investissements intellectuels réguliers.

Lorsque la PME intègre en son sein l'ensemble des activités "primaires » ou «de soutien», au sens de Porter (1985), la mobilisation de ressources intellectuelles sera plus importante que lorsque cette entreprise limite son activité à la fabrication (en général, par contrat de sous-traitance). Dans la première situation, la PME élabore une stratégie pro-active.

Hypothèse 3 : Les PME qui réalisent des investissements matériels, intellectuels et organisationnels intégrés auront plus facilement accès à des marchés extérieurs.

On distinguera alors le processus de «maturation provoquée» dans le cas d'une stratégie anticipative et le processus de «maturation contrainte» dans le cas d'une stratégie adaptative (Guilhon, 1993).

On peut supposer que la PME qui s'attaque à des marchés plus concurrentiels ou qui développe ses activités sur plusieurs marchés à la fois devra mobiliser plus de ressources et de ce fait aura une attitude plus énergique.

Hypothèse 4: La logique ou l'appartenance sectorielle n'est pas déterminante pour expliquer les comportements d'entreprise.

L'acte d'exportation relève de l'attitude individuelle du dirigeant (perception de l'environnement, prise de décision, mise en œuvre stratégique).

\subsection{Méthodologie}

L'analyse présentée ci-dessous se fonde sur une enquête effectuée auprès d'entreprises de la région PACA au cours du premier semestre 1993. 
L'échantillon a été constitué à partir des fichiers de la CCIMP et comportait au départ 276 entreprises ayant un effectif compris entre 10 et 500 employés. Le nombre de réponses obtenues a été de 108, dont 100 ont pu être exploitées. Cela correspond à un taux de réponse de $36 \%$.

Les entreprises ont été choisies en fonction de leur appartenance sectorielle. Les secteurs ciblés pour cette étude ont été ceux de la nomenclature NAP 100 compris entre 20 et 29, c'est-à-dire les secteurs de production de biens d'équipement et de production industrielle. Certaines entreprises (5) classées dans ces secteurs, d'après les fichiers de la CCIMP, se sont révélées avoir leur activité principale dans un autre secteur. Elles ont été exclues par la suite des analyses effectuées.

La répartition des entreprises ayant répondu au questionnaire est présentée dans le tableau 1.

Le questionnaire comporte 11 chapitres permettant de fournir des valeurs à 67 variables.

1. Implantation de nouvelles techniques (INT dans la suite)

2. Formation après les implantations

3. Formation avant les implantations

4. Emploi dans l'entreprise

5. Actions de R-D

6. Actions de communication

7. Embauche récente

8. Raisons ayant conduit à l'implantation technique

9. Chiffre d'affaires

10. Sous-traitance

11. Exportation

Lorsque des données chiffrées étaient demandées, elles portaient sur deux années, 1987 et 1992.

Parallèlement, un traitement statistique a été réalisé sur les données du commerce extérieur de la région PACA. Fournies par le Service statistique des Douanes de Toulouse, ces données portent sur deux années, 1983 et 1990. Des indicateurs ont été construits, tel l'indice de spécialisation sectorielle:

$$
S_{1}=\begin{aligned}
& \text { Exportations - Importations } \\
& \text { Exportations + Importations }
\end{aligned}
$$


Le tableau 1 indique que les PME investissent dans des nouvelles techniques plus ou moins intégrées, indépendamment de l'évolution de la spécialisation internationale de leur secteur d'appartenance (voir les secteurs 2106 et 2408). La stratégie d'investissement matériel (hypothèse 1) semble correspondre à une volonté d'engagement sur les marchés extérieurs.

TABLEAU 1

Répartition des entreprises par secteur et spécialisation internationale

\begin{tabular}{|c|c|c|c|c|c|c|}
\hline \multirow[t]{2}{*}{$\begin{array}{l}\text { Code } \\
\text { APE }\end{array}$} & \multirow[t]{2}{*}{$\begin{array}{c}\text { Nombre } \\
\text { d'entreprises }\end{array}$} & \multirow{2}{*}{$\begin{array}{c}\text { Entreprises } \\
\text { ayant } \\
\text { implanté } \\
\text { des NT }\end{array}$} & \multirow[t]{2}{*}{$\begin{array}{c}\text { INT } \\
\text { faibles }\end{array}$} & \multirow[t]{2}{*}{$\begin{array}{l}\text { INT } \\
\text { fortes }\end{array}$} & \multicolumn{2}{|c|}{$\begin{array}{l}\text { Spécialisation } \\
\text { internationale }\end{array}$} \\
\hline & & & & & 1983 & 1990 \\
\hline 1101 & 2 & 2 & 1 & 1 & N.D. & N.D. \\
\hline 1105 & 1 & 1 & 0 & 1 & N.D. & N.D. \\
\hline 2102 & 2 & 2 & 0 & 2 & 64,4 & $-12,2$ \\
\hline 2103 & 3 & 2 & 1 & 1 & N.D. & N.D. \\
\hline 2106 & 5 & 5 & 2 & 3 & 39,8 & $-25,8$ \\
\hline 2108 & 26 & 22 & 15 & 7 & 16,5 & $-11,1$ \\
\hline 2114 & 1 & 1 & 0 & 1 & $-15,4$ & $-21,6$ \\
\hline 2115 & 2 & 1 & 1 & 0 & $-43,7$ & 78,1 \\
\hline 2301 & 2 & 1 & 1 & 0 & 2,67 & $-2,82$ \\
\hline 2303 & 1 & 1 & 1 & 0 & $-30,9$ & $-47,2$ \\
\hline 2401 & 2 & 2 & 0 & 2 & $-4,8$ & $-1,8$ \\
\hline 2403 & 11 & 9 & 4 & 5 & $-13,2$ & $-33,7$ \\
\hline 2404 & 1 & 1 & 1 & 0 & 62,5 & 54,9 \\
\hline 2408 & 28 & 26 & 19 & 7 & 43,9 & 65,3 \\
\hline 2409 & 4 & 4 & 0 & 4 & $-3,9$ & $-25,5$ \\
\hline 2811 & 4 & 4 & 4 & 0 & 21,45 & $-11,7$ \\
\hline 2815 & 1 & 1 & 0 & 1 & N.D. & N.D. \\
\hline 2913 & 2 & 2 & 1 & 1 & 10.54 & 14,0 \\
\hline 5302 & 2 & 2 & 1 & 1 & N.D. & N.D. \\
\hline & Total $=100$ & 89 & 52 & 37 & & \\
\hline
\end{tabular}

Note: INT fortes correspondent à des implantations simultanées de machines-outils à commande numérique, de CAO-DAO, GPAO et FAO, c'est-à-dire qui concernent un ensemble de postes et de fonctions dans l'entreprise. INT faibles correspondent à des implantations de machines-outils à commande manuelle, de micro-informatique qui concernent un seul poste de travail. 
Pour le traitement des données de l'enquête, nous avons utilisé des méthodes classiques d'analyse de données (analyse factorielle des correspondances, analyse hiérarchique, tri croisé).

\section{Résultats et interprétations}

Nous procéderons en trois étapes :

\subsection{L'énoncé des six dichotomies et la construction des profils d'évolution}

\subsubsection{Présentation}

Le tableau 2 présente la répartition des entreprises par groupes constitués à partir des analyses de données complétées par des analyses hiérarchiques. Les groupes sont mis en relation avec les six premières variables du questionnaire, après regroupement des deux variables de formation.

La variable implantation des nouvelles techniques est caractérisée comme suit :

- L'implantation de nouvelles techniques: le changement technologique intervient de deux façons: sur le mode intégré (GPAO, FAO, CAO/ DAO...) et non intégré (seuls certains postes de travail sont concernés : MOCN, MOCM, micro-informatique)

TABLEAU 2

Typologies des comportements d'investissement dans les PME étudiées

\begin{tabular}{|c|c|c|c|}
\hline Chapitre & $\begin{array}{l}\text { Pourcentage } \\
\text { d'entreprises } \\
\text { concernées }\end{array}$ & Groupe 1 & Groupe 2 \\
\hline $\begin{array}{l}\text { Implantation } \\
\text { technique }\end{array}$ & 89 & $\begin{array}{l}\text { Faiblement intégrée } \\
\text { (MOCM, MOCN, } \\
\text { micro-informatique) }\end{array}$ & $\begin{array}{l}\text { Fortement intégrée } \\
\text { (MOCN + GPAO + } \\
\text { CAO/DAO + FAO) }\end{array}$ \\
\hline Formation & 83 & Formation courte & Formation longue \\
\hline Emploi & 100 & $\begin{array}{l}\text { Production - } \\
\text { encadrement }\end{array}$ & $\begin{array}{l}\text { Conception - } \\
\text { production }\end{array}$ \\
\hline R-D & 54 & Procédés & Produits - procédés \\
\hline Embauche & 71 & $\begin{array}{l}\text { Ingénieurs - } \\
\text { techniciens }\end{array}$ & $\begin{array}{l}\text { Commerciaux - } \\
\text { Comptables }\end{array}$ \\
\hline Communication & 79 & Publicité & $\begin{array}{l}\text { Études de marché - } \\
\text { publicité - conseil externe }\end{array}$ \\
\hline
\end{tabular}


La discrimination est ensuite analysée, pour les PME ayant implanté des nouvelles techniques, à partir de chaque rubrique et de la manière suivante:

- La formation: les stages de formation ont lieu après l'introduction des nouvelles techniques; ils sont ponctuels et de courte durée (moins de trois mois). Ils concernent essentiellement les employés et se réalisent à l'extérieur de l'entreprise. Pour certaines entreprises, les stages de formation se déclenchent avant les implantations techniques et concernent les employés, le personnel clé et le dirigeant, et ils ont souvent lieu dans l'entreprise.

- L'emploi: la dichotomie entre les PME s'effectue sur les activités de fabrication et de conception. On observe que les PME sont structurées principalement en fonction d'une activité de fabrication et présentent un nombre important d'individus à l'encadrement, alors que celles qui sont structurées en fonction d'une activité de conception et de fabrication ont un nombre important d'individus à l'administration.

- L'embauche: la dichotomie entre les PME sur l'embauche est réalisée suivant la nature des «spécialités» des individus. Soit qu'elles aient embauché des ingénieurs et des informaticiens, c'est-à-dire des «généralistes » des techniques, et n'aient pas embauché d'autres catégories d'individus, soit qu'elles n'aient pas embauché ces techniciens mais principalement des commerciaux et des comptables.

- La R-D: les PME, réalisant de la R-D seulement sur les procédés, n'ont recours à cette activité que ponctuellement, principalement pour la programmation des plans et des cotes. En revanche, les PME réalisant de la $\mathrm{R}-\mathrm{D}$ sur les produits et les procédés ont recours à cette activité régulièrement et depuis longtemps (plus de cinq ans au moment de l'étude).

- La communication: la dichotomie pour la communication révèle le même scénario que pour la R-D. Elle s'effectue sur la régularité et la nature de la communication. Ainsi, on remarque que les PME réalisant uniquement de la publicité le font ponctuellement, alors que celles qui réalisent des études de marché, de la publicité et qui ont recours à des conseils, le font régulièrement. On peut voir ici que les PME se divisent entre celles qui désirent produire ce qu'elles peuvent vendre et celles qui veulent vendre ce qu'elles «savent» produire.

\subsubsection{Analyse de la relation entre implantation} de nouvelles techniques (INT) et chacun des autres thèmes

- L'INT et la formation: la discrimination des PME s'établit sur la durée des stages de formation après les INT, d'une part (en relation avec l'existence de stages de formation avant ces implantations), et sur le degré d'implantation des techniques, d'autre part. 
On constate que les PME ayant implanté des nouvelles techniques peu intégrées ont organisé des stages de formation de plus de trois mois pour leurs employés, mais après ces implantations.

Les PME ayant implanté des nouvelles techniques très intégrées ont organisé des stages de formation de moins de trois mois après ces implantations, mais ont toutes réalisé des stages de formation pour les employés, le dirigeant et le personnel clé avant les implantations.

On remarque donc que le besoin d'apprentissage est différent, après les nouvelles techniques, suivant l'existence de la formation avant ces implantations.

- L'INT et l'embauche: l'embauche est fortement reliée aux INT. Plus les implantations sont intégrées et plus les entreprises ont embauché, notamment des ingénieurs et des informaticiens. Mais si les nouvelles techniques sont peu intégrées, l'embauche concerne uniquement un poste (technicien spécialisé : opérateur numérique, cisailleur...) ou des commerciaux et des comptables. Cette dichotomie reflète bien la spécialisation des PME qui investissent dans un objectif d'innovation ou dans un objectif de valorisation des ressources existantes.

- L'INT et l'emploi: il est intéressant de voir que la structure d'emploi met en évidence la spécialisation et les orientations dans les activités des PME. Les PME qui ont investi dans des nouvelles techniques très intégrées sont structurées suivant une dominance de conception, de fabrication et d'administration. Les PME ayant implanté des nouvelles techniques peu intégrées, c'est-à-dire sur un ou plusieurs postes (MOCN, MOCM) sont structurées suivant une dominance de production et d'encadrement, ce qui est parfaitement compréhensible dans la mesure où les implantations concernent uniquement des postes de fabrication.

- L'INT et la R-D: l'analyse des correspondances montre ici deux choses: les oppositions entre les PME se font d'après l'existence de la R-D avant et après les INT, mais aussi suivant le degré d'INT. Les PME qui effectuent de la R-D sur les produits et sur les procédés depuis plus de trois ans sont celles qui ont investi massivement dans des techniques très intégrées. En revanche, les PME qui ont investi dans des nouvelles techniques peu intégrées ne font pas de R-D ou en font seulement sur les procédés.

- L'INT et la communication: comme pour la R-D, ce sont l'existence et la durée de la communication suivant le degré d'implantation technique qui sont discriminantes. Les PME, réalisant de la communication 
régulière (étude de marché, publicité et conseil), sont celles qui ont investi dans des nouvelles techniques très intégrées. Alors que les PME qui ont implanté des nouvelles techniques peu intégrées ont réalisé principalement de la publicité ponctuelle.

Les résultats des analyses permettent d'identifier des profils de PME en fonction du degré d'implantation des nouvelles techniques et des investissements intellectuels plus ou moins réguliers qu'elles ont développés. On s'aperçoit donc que l'innovation technologique et organisationnelle dépend d'une stratégie de positionnement sur les marchés, axée sur la recherche d'innovation ou sur le maintien d'une activité existante. L'identification de ces profils permet de vérifier la seconde hypothèse.

\subsection{Synthèse des résultats}

Elle est obtenue à partir d'un échantillon de $46 \mathrm{PME}$ ayant réalisé des implantations significatives en matériel de commandes numériques et de commandes manuelles. Ces implantations sont considérées comme la condition indispensable à la mise en place de dispositifs techniques plus intégrés (graphique 1).

La représentation simultanée, à l'aide de l'analyse factorielle des correspondances, fait apparaître une contribution totale des axes à l'inertie du nuage de $32,9 \%$. C'est un résultat faible qui s'explique par le poids des variables dans la représentation: les profils des PME représentées concentrent la plupart des variables du plan factoriel dans la mesure où n'ont été retenues que les PME ayant réalisé des implantations de MOCN et de MOCM et étant susceptibles d'avoir réalisé des investissements intellectuels.

L'axe 1 est celui de la structure d'emploi, le long duquel s'opposent les PME ayant une structure dominée par la production (droite) et celles dominées par la conception (gauche). Sur cet axe évoluent les investissements intellectuels réalisés après les INT.

L'axe 2 est celui du degré d'INT, le long duquel s'opposent les PME ayant implanté des techniques de plus en plus complexes et systémiques.

Le plan factoriel $1 * 2$ fait donc apparaître des profils de PME en opposition sur les axes: les PME sont discriminées par leur positionnement sur la chaîne de valeur (production, conception, commercialisation) en fonction des implantations techniques qu'elles ont réalisées.

Plus les PME sont centrées sur l'activité de production, plus les INT sont autonomes (sur un poste), et plus les investissements intellectuels dispensés sont essentiellement de la formation ponctuelle avec l'embauche de commerciaux, comptables ou techniciens spécialisés. 
En évoluant le long de l'axe 1 vers la gauche et le long de l'axe 2 vers le bas, on repère des PME dont la structure est centrée sur la conceptionfabrication et qui ont implanté des nouvelles techniques de plus en plus intégrées suivant les investissements intellectuels réalisés: GPAO, puis CAO et INT systémiques intégrées. Les investissements intellectuels sont réguliers avant les INT et concernent en premier lieu la R-D et la communication. Ensuite, ils prennent un caractère systémique.

\section{GRAPHIQUE 1 \\ Analyse des données globales}

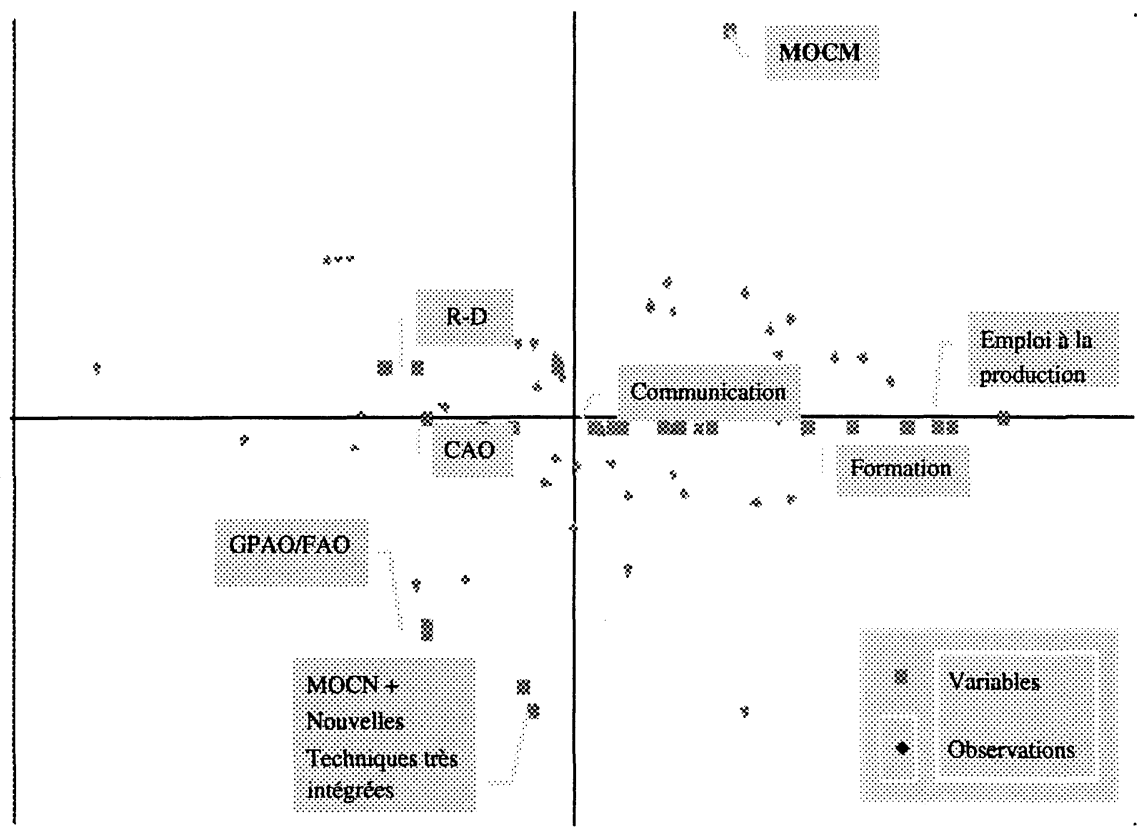

Note: Ce graphique s'interprète en termes de proximités : la distance d'un point représentant une observation à un point représentant une variable mesure l'importance de cette variable pour la PME (observation) concernée.

La contribution relative des points aux axes montre, d'une part, la forte correspondance entre la formation et l'embauche et, d'autre part, la forte correspondance entre la R-D et la communication régulière.

Il y a donc une évolution des techniques en parallèle avec l'évolution des compétences. 


\subsection{La compatibilité entre l'innovation et l'exportation: la réorganisation d'ensemble de l'entreprise}

L'échantillon analysé est sans doute trop restreint pour tirer des conclusions générales sur les liens qui peuvent exister entre le développement de l'exportation et la réorganisation de l'entreprise du fait de l'implantation de nouvelles techniques. Cependant, certaines remarques «locales» peuvent être formulées.

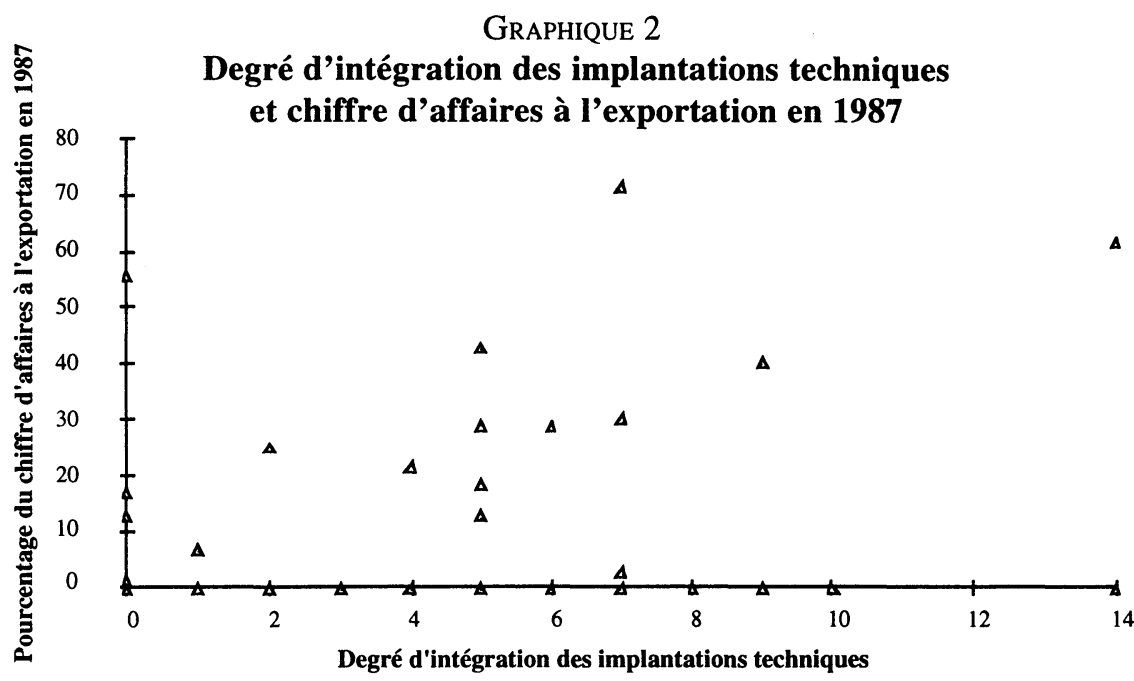

GRAPHIQUE 3

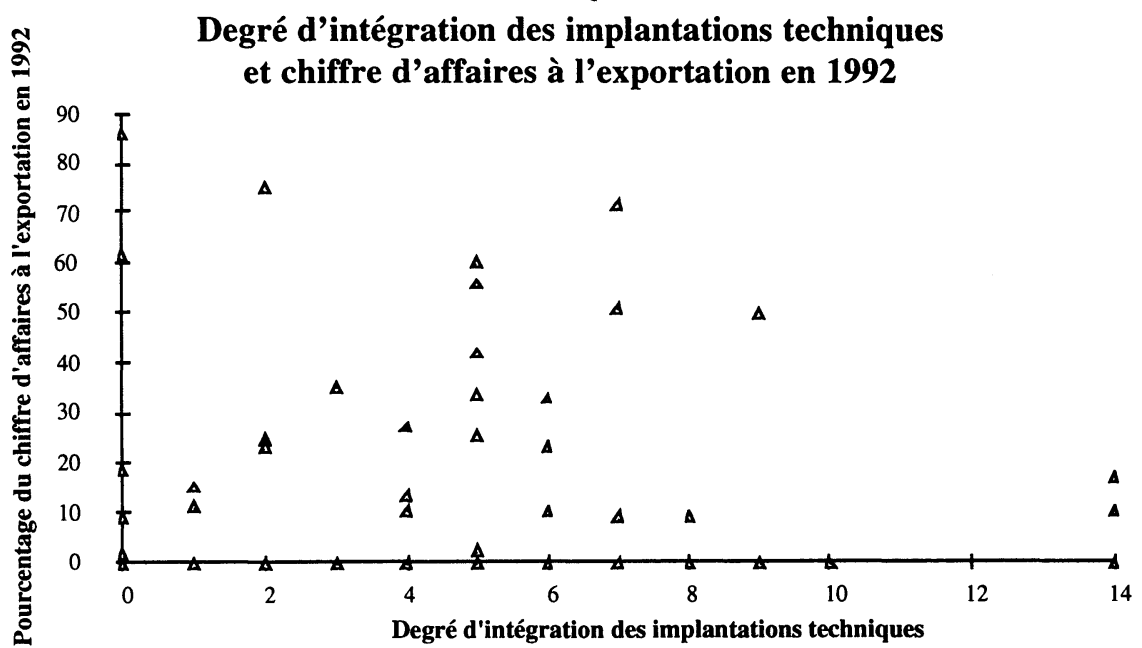


1. Les graphiques 2 et 3, qui représentent pour 1987 et 1992 la relation entre la part du chiffre d'affaires à l'exportation et un index d'intégration de nouvelles techniques ${ }^{2}$, autorisent deux remarques.

- Tout d'abord, la situation de départ (en 1987, graphique 2) a une importance non négligeable. Il semble bien que plus les entreprises sont engagées à l'exportation à cette date, plus elles effectuent des implantations techniques intégrées. L'explication peut provenir du fait que la présence plus forte sur des marchés étrangers soumet la firme à une concurrence plus intense, la poussant alors à rechercher une efficacité plus grande (Bonaccorsi, 1992).

- Ensuite, la situation d'arrivée (en 1992, graphique 3) est en apparence paradoxale. Nous pouvions penser que l'implantation de nouvelles techniques intégrées aurait renforcé la présence des firmes sur les marchés étrangers. Il n'en est rien. Tout se passe comme si l'implantation intégrée de nouvelles techniques avait permis à l'entreprise de se recentrer sur son marché national en stabilisant ses marchés extérieurs (existence d'un seuil autour des valeurs 6-7 du degré d'implantation). Ce tableau tend à montrer que la relation entre l'investissement en nouvelles techniques et le pourcentage du chiffre d'affaires réalisé à l'exportation n'est pas directe, mais médiatisé par la stratégie adoptée par le dirigeant (Roux, 1991).

2. En évolution (graphique 4), nous observons que les entreprises qui ont implanté des nouvelles techniques n'ont pas enregistré d'évolution marquée de leur chiffre d'affaires. Nous pouvons émettre l'hypothèse que ces PME n'ont pas poursuivi leur effort à l'exportation.

3. Les graphiques 5 et 6 semblent remettre en cause l'idée reçue selon laquelle l'effort d'exportation est lié à la taille de l'entreprise. Selon le graphique 6 , ce sont les entreprises ayant un effectif faible qui ont une

2. Cet index a été construit à partir des réponses au questionnaire en pondérant chaque type d'implantation technique. En l'occurrence, les pondérations utilisées sont :

$\begin{array}{ll}\text { - Implantation de MOCN } & 5 \\ \text { - GPAO } & 4 \\ \text { - CAO } & 3 \\ \text { - DAO } & 2 \\ \text { - MOCM } & 1\end{array}$

De telle sorte qu'une entreprise ayant effectué une implantation fortement intégrée (MOCN + GPAO + CAO + DAO) obtient un index égal à 14, alors qu'une entreprise ayant implanté seulement une MOCM a un index égal à 1 . 
activité d'exportation (en proportion du chiffre d'affaires) plus importante. En évolution, il semble même que ce soit les plus grandes PME qui aient réduit le plus leur activité d'exportation entre 1987 et 1992 (Bonaccorsi, 1992). Dans les deux cas, on note l'existence d'un effet de seuil situé autour de 50 salariés qui peut être interprété de deux façons:

- les investissements intellectuels réalisés dans les PME sont trop ponctuels et ne permettent pas d'accéder à des ressources externes indispensables (informations sur les marchés et les produits, construction de relations stables entre acheteurs et vendeurs, politique de communication...);

- la poursuite de l'objectif d'exportation exige des adaptations des ressources et de l'organisation qui semblent incompatibles avec la capacité du dirigeant à contrôler cette évolution et à conduire ces transformations. Dans ce cas, la volonté de pérennisation l'emporte sur la volonté de croissance.

GRAPHIQUE 4

Degré d'intégration des implantations techniques et évolution du chiffre d'affaires en pourcentage de 1987 à 1992

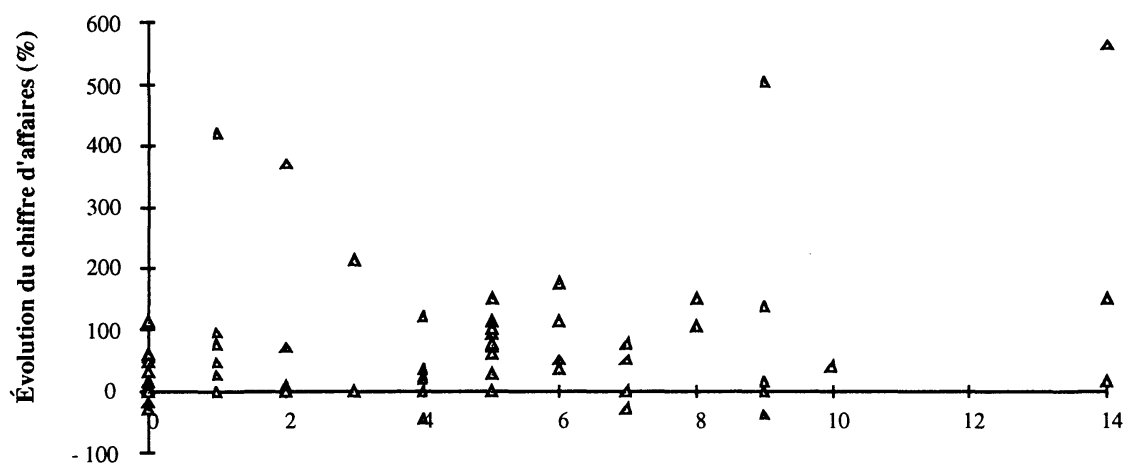

Degré d'intégration des implantations techniques 
GRAPHIQUE 5

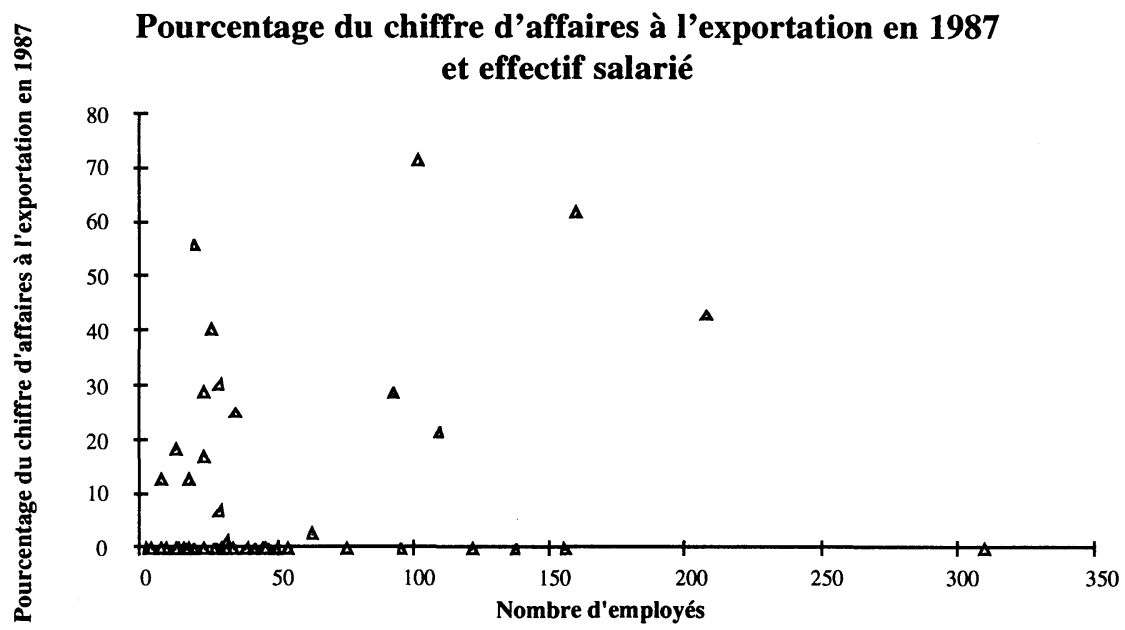

GRAPHIQUE 6

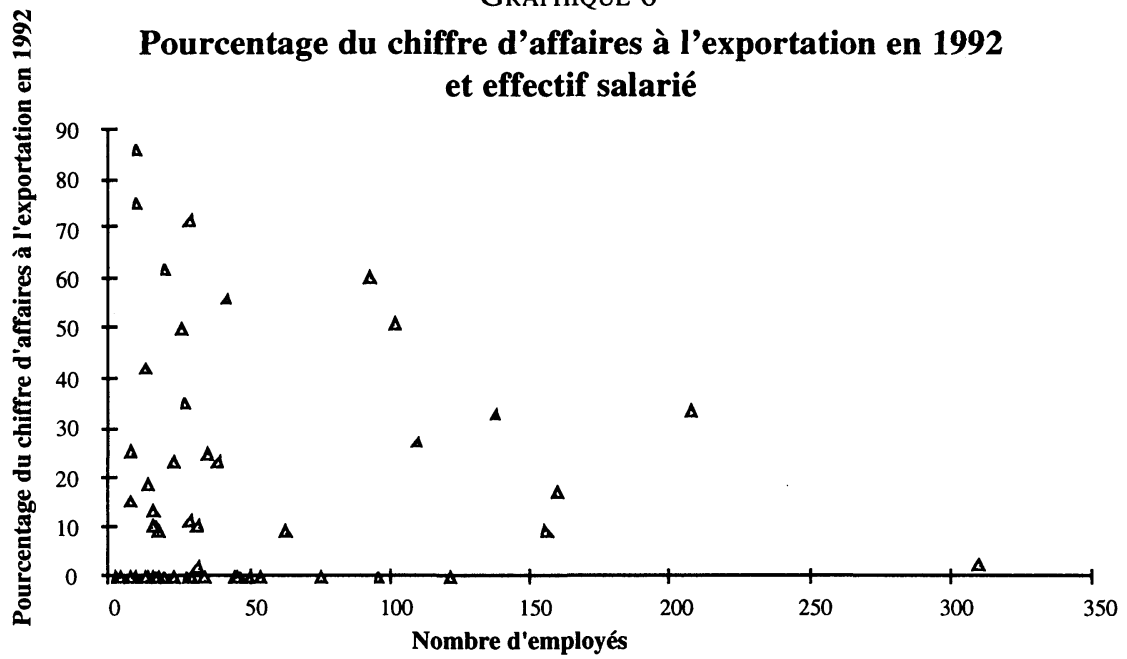




\section{Conclusion}

L'étude menée sur l'échantillon retenu souligne l'existence de deux stratégies centrées sur le rôle du dirigeant pour atteindre les marchés extérieurs. La stratégie anticipative repose sur la transformation des ressources internes et l'accès à des marchés extérieurs. La stratégie adaptative indique que le positionnement sur des marchés extérieurs se réalise par des adaptations contraintes et donc plus ponctuelles. On constate également que l'exportation devient compatible avec l'innovation dans les PME lorsque deux conditions sont satisfaites:

1. Le développement des compétences appréciées par les investissements intellectuels et la réorganisation d'ensemble des entreprises. Un schéma récursif se manifeste comme suit:

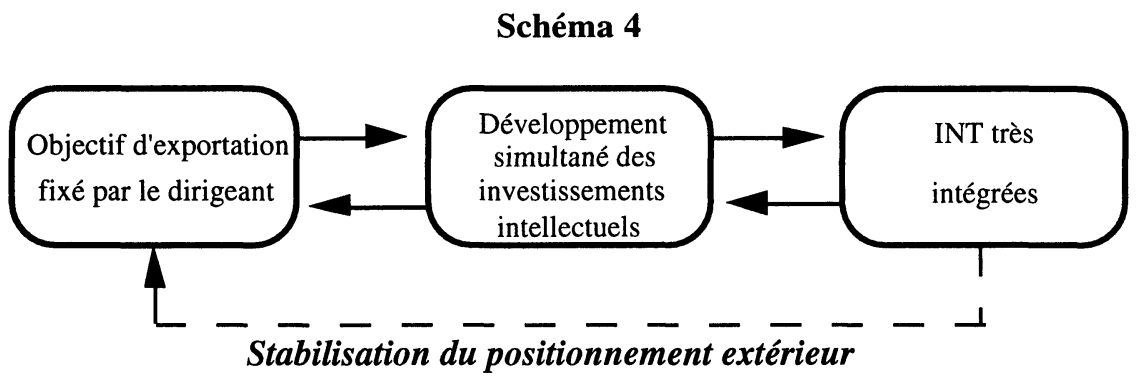

L'analyse proposée remet en cause l'influence de la taille ou de l'appartenance sectorielle comme déterminants de l'engagement à l'exportation et souligne l'existence d'un effet de seuil autour de 50 salariés.

2. Que le processus de maturation soit contraint ou provoqué, la compatibilité entre l'exportation et l'innovation traduit le rôle central que joue le dirigeant de la PME.

\section{Bibliographie}

AMmeuX, J.P. (1984), Les Petites et moyennes entreprises en France, Thèse de troisième cycle, Paris I-Panthéon Sorbonne.

BONACCORSI, A. (1992), «On the relationship between firm size and export intensity », Journal of International Business Studies, vol. 32.

Bouchet, H. (1989) «L'investissement intellectuel», Conseil Économique et Social, Journal Officiel de la République Française. 
BRICOUT, J.L. (1991), «Exporter, une affaire de taille», Économie et Statistiques.

Chicha, J., P.A. Julien et M. MARChesnay (1990), «Processus et procédures stratégiques dans les PME », Internationales Gewebearchiv, $\mathrm{n}^{\circ} 3$.

GuILHON, A. (1993), Étude de la relation entre le changement organisationnel et l'investissement intellectuel dans les PME, Thèse de doctorat Nouveau Régime, Université de Montpellier I.

JULIEN, P.A. et R. JACOB (1993), «Les nouvelles technologies», dans PME: bilan et perspectives, Paris, Économica et Québec, Les Presses Inter Universitaires.

KALIKA, M. (1991), «De l'organisation réactive à l'organisation anticipative», Revue Française de Gestion, ${ }^{\circ}$ spécial 86.

MARCheSnay, M. (1990), «La PME: une gestion spécifique?», Colloque de la Société Française d'Économie Rurale.

Marchesnay, M. et P.A. Julien (1988), «La petite entreprise », Paris, Vuibert Gestion.

MARCHESNAY, M. (1993), «Le management stratégique», dans Les PME: Bilan et perspectives, op. cit., chap. 5.

MoINI, A.H. (1992), « Europe 1992: a challenge to small exporters », Journal of Small Business Management, vol. 30, $\mathrm{n}^{\circ} 1$.

PORTER, M. (1985), «Choix stratégique et concurrence », Paris, InterÉdition.

Roux, E. (1991), Les facteurs explicatifs de la décision d'exporter en PMI; le rôle et l'attitude du dirigeant envers le risque, Thèse de doctorat d'État, Université de Montpellier.

SESSI (1990), PMI : chiffres clés, Paris, Dunod.

SESSI (1992), Rapport du service des statistiques industrielles.

WALTERS, P. et S. SAMLEE (1990), «A model for assessing performance in small U.S exporting firms », Entrepreneurship Theory and Practice, vol. 15, $\mathrm{n}^{\circ} 2$. 